\title{
Therapeutic plasma exchange in patients in a portuguese ICU
}

\author{
Rita Calça ${ }^{1}$, Ana Gaspar ${ }^{2}$, Afonso Santos ${ }^{2}$, Adelina Aufico ${ }^{3}$, Paulo Freitas ${ }^{3}$, Sílvia Coelho ${ }^{3}$ \\ ${ }^{1}$ Nephrology Department, Hospital de Santa Cruz - Centro Hospitalar de Lisboa Ocidental, Carnaxide, Portugal \\ 2 Nephrology Department, Hospital Prof. Doutor Fernando Fonseca, Amadora, Portugal \\ ${ }^{3}$ Intensive Medicine Department, Hospital Prof. Doutor Fernando Fonseca, Amadora, Portugal
}

\section{ABSTRACT}

Purpose: The aim of this study is to characterize a Portuguese Intensive Care Unit experience in therapeutic plasma exchange in critically ill patients. Methods: We performed a retrospective analysis of the patients treated with therapeutic plasma exchange between 2000 and 2019. Data on patient characteristics, therapeutic plasma exchange prescription, adjuvant therapy used, adverse events and outcome under treatment were collected. Results: A total of 101 therapeutic plasma exchange procedures in 20 patients were studied. Mean number of therapeutic plasma exchange sessions per patient was $5.1 \pm 1.3$. The most frequent indications to begin this treatment were myasthenia gravis (25.0\%) and anti-neutrophil-cytoplasmic antibody-associated vasculitis (15.0\%). There were $45.5 \%$ adverse events and the most frequent was hypotension (15.2\%). $98 \%$ of the complications were mild-to-moderate. The outcome was favorable in $60.0 \%$ of patients. Conclusion: Therapeutic plasma exchange is an effective and safe therapy in many diseases that had high morbidity and mortality prior to the use of this technique.

Keywords: apheresis, adverse events, critical care, plasmapheresis, therapeutic plasma exchange.

\section{INTRODUCTION}

Therapeutic plasma exchange (TPE) is an extracorporeal technique in which plasma is separated from the remaining blood components and exchanged by a replacement fluid, usually fresh frozen plasma (FFP) or albumin, depending on the disease, coagulation and immune status $^{1-3}$. Separation of plasma components can be performed through centrifugal apheresis or filtration ${ }^{1,2,4,5}$.

The main mechanism of action of TPE is the rapid removal of specific pathogens, such as abnormal antibodies, paraproteins, antigen-antibody complexes, alloantibodies, endogenous toxins or exogenous poisons ${ }^{1,4}$. Another important role of TPE is the replacement of the blood plasma with normal FFP in patients with deficiency of a plasma component, for instances, in patients with thrombotic thrombocytopenic purpura (TTP) and ADAMTS 13 deficit $^{2}$.

In 2019, the American Society for Apheresis (ASFA) published the most recently updated guidelines for the use of therapeutic apheresis in clinical practice $2,6,7$, many of them affecting critically ill patients.

TPE is a generally safe and well-tolerated technique but adverse events exist ${ }^{1}$ and they are a particular concern in the critical care setting, where the margin of error is very narrow. Critically ill patients are more likely to experience hemodynamic instability, coagulation disorders or electrolyte disturbances that may hamper the efficacy of the technique ${ }^{8}$. The reported complication rate is $4-25 \%{ }^{2}$ and the incidence of complications depends on the anticoagulation and replacement fluid used, volume exchanged, vascular access, plasma separation method and underlying disease ${ }^{9}$. The most common adverse effects are paresthesias, muscle cramps, hypotension, and urticaria $^{1,9}$. Most complications are mild or moderate ${ }^{1,2}$ and severe complications that require interruption of the TPE treatment represent only $0.8 \%^{1,10}$. Severe complications are usually caused by anaphylactic reactions to the replacement fluid and are most common with FFP 1,2 . The rate of complications appears to be similar in patients admitted to the Intensive Care Unit (ICU), although data is much scarcer ${ }^{8,11}$.

The aim of the study is to characterize the use of TPE in critical care patients, with a focus on the indications, technical aspects of the technique prescription, complications and outcomes. This is the first study to characterize the use of TPE in a Portuguese ICU.

\section{PATIENTS AND METHODS}

We performed a retrospective analysis of all adult patients submitted to TPE at the Intensive Medicine Department (IMD) of Fernando Fonseca Hospital, Lisbon, Portugal, between January 2000 and April 2019. No exclusion criteria were applied. Fernando Fonseca Hospital is a public hospital with 802 beds and its IMD is composed of 1 mixed surgical and medical ICU and 1 surgical ICU, with a total of 16 level III and 4 level II beds. Our institutional ethics committee approved the study and informed consent was waived due to the retrospective nature of the study.

Indications to initiate TPE treatment were based on ASFA guidelines ${ }^{6}$. Number, frequency and exchanged plasma volumes depended on the underlying disease and clinical outcome. Estimated plasma volume for each treatment was calculated with the formula: $0.07 \mathrm{x}$ 
weight $(\mathrm{kg}) \times(1-\text { hematocrit })^{1}$. TPE treatments were performed using the membrane separation technique with Prismaflex ${ }^{\circledR}$, Gambro ${ }^{\circledR}$ (Sweden) equipment using hemodialysis catheters of dual lumen.

Data on demographic characteristics, hospitalization days, underlying disease, Acute Physiology and Chronic Health Evaluation (APACHE II) and Simplified Acute Physiology Score (SAPS II) scores on admission to ICU, TPE prescription parameters (number of procedures performed, replacement fluid and anticoagulation used), adjuvant treatments (immunosuppressive treatment, renal replacement therapy (RRT), mechanical ventilation, vasopressor support and antibiotic therapy use), adverse events related to the technique and patients' outcome under treatment, were collected.

The following complications were documented: presence of symptoms such as nausea or vomiting, paresthesia, muscle cramps; hypotension (defined as $20 \%$ decrease from baseline values of systolic blood pressure); infection; bleeding; laboratory abnormalities such as anemia (hemoglobin $<12 \mathrm{~g} / \mathrm{dl}$ ); hyponatremia (serum sodium $<135 \mathrm{mEq} / \mathrm{L}$ ); hypokalemia (serum potassium $<3.5 \mathrm{mEq} / \mathrm{L}$ ) and hypocalcemia (ionized serum calcium $<2.1 \mathrm{mmol} / \mathrm{L}$ ). Moreover, adverse events were classified according to their severity: (I) mild, no need for therapeutic measures; (II) moderate, signs and symptoms during treatment that required therapeutic intervention but without interruption of the treatment; (III) severe, if the adverse event forced the interruption of the treatment.

The outcome was defined as favorable if there was an improvement in clinical and laboratory parameters and unfavorable in case of worsening of clinical status under TPE.

Descriptive analysis was performed using the IBM ${ }^{\circledR}$ SPSS ${ }^{\circledR}$ Statistics software for Windows, version 22. Categorical variables are presented as frequency distributions or percentages. Continuous variables are presented as mean \pm standard deviation (SD) or median and interquartile range (IQR) [25th-75th percentile (P25 to P75)] if they were not normally distributed. The normality of the distribution was assessed using the Kolmogorov-Smirnov test.

\section{RESULTS}

\section{Patient characteristics}

This cohort includes 20 patients submitted to 101 TPE procedures in a single ICU. The mean age was $44.3 \pm 19.9$ years-old (range: $20-83$ years) and $60 \%$ were female.

The hospital length of stay (LOS) was 32.0 (15.0-69.0) days and the ICU LOS was 10.0 (7.0-30.2) days. The mean SAPS II was $28.9 \pm 14.7$ and the mean APACHE II was $12.8 \pm 6.9$. Description of the cohort is summarized in Table I.

The indication for TPE was myasthenia gravis in $24.8 \%$ of the procedures; anti-neutrophil cytoplasmic antibody (ANCA) associated vasculitis with RPGN and/or alveolar hemorrhage in 14.9\%; immune complex rapidly progressive glomerulonephritis (RPGN) in $11.9 \%$; TTP in 11.9\%; anti-N-methyl D-aspartate (NMDA) receptor antibody

\section{Table I}

Characteristics of the study patients

\begin{tabular}{|c|c|}
\hline \multicolumn{2}{|l|}{ Demographics } \\
\hline Age (years $\pm S D$ ) & $44.3 \pm 19.9$ \\
\hline Female gender ( $n, \%)$ & $12,60.0 \%$ \\
\hline SAPS II (mean \pm SD) & $28.9 \pm 14.7$ \\
\hline APACHE II (mean \pm SD) & $12.8 \pm 6.9$ \\
\hline \multicolumn{2}{|c|}{ Indications for plasma exchange therapy } \\
\hline \multicolumn{2}{|l|}{ Neurologic } \\
\hline Myasthenia gravis (n, \%) & $5(25.0 \%)$ \\
\hline NMDA receptor antibody encephalitis (n, \%) & $2(10.0 \%)$ \\
\hline Guillain-Barré syndrome (n, \%) & $1(5.0 \%)$ \\
\hline Acute polyradiculoneuropathy Guillain-Barré like (n, \%) & $1(5.0 \%)$ \\
\hline Chronic demyelinating polyradiculoneuropathy (n, \%) & $1(5.0 \%)$ \\
\hline \multicolumn{2}{|l|}{ Renal } \\
\hline ANCA-associated vasculitis with RPGN and/or alveolar & \\
\hline $\begin{array}{l}\text { hemorrhage }(n, \%) \\
\text { Immune complex RPGN (n, \%) }\end{array}$ & $\begin{array}{l}3(15.0 \%) \\
2(10.0 \%)\end{array}$ \\
\hline \multicolumn{2}{|l|}{ Thrombotic microangiopathy } \\
\hline $\operatorname{TTP}(\mathrm{n}, \%)$ & $2(10.0 \%)$ \\
\hline HUS (n, \%) & $2(10.0 \%)$ \\
\hline \multicolumn{2}{|l|}{ Endocrine } \\
\hline Thyrotoxic crisis (n, \%) & $1(5.0 \%)$ \\
\hline \multicolumn{2}{|l|}{ Organ support therapies } \\
\hline Vasopressor support (n, \%) & $3(15.0 \%)$ \\
\hline Invasive mechanical ventilation ( $\mathrm{n}, \%$ ) & $10(50.0 \%)$ \\
\hline Renal replacement therapy (n, \%) & $7(35.0 \%)$ \\
\hline \multicolumn{2}{|l|}{ Outcome data } \\
\hline UCI length of stay (median days [IQR]) & $10.0[7.0-30.0]$ \\
\hline Hospital length of stay (median days [IQR]) & $32.0[15.0-69.0]$ \\
\hline Mortality & $2(10.0 \%)$ \\
\hline Favorable outcome & $12(60.0 \%)$ \\
\hline \multicolumn{2}{|c|}{$\begin{array}{l}\text { ANCA - anti-neutrophil cytoplasmic antibody; HUS - hemolytic-uremic syndrome; TTP - thromboti } \\
\text { thrombocytopenic purpura; NMDA - anti-N-methyl D-aspartate; RPGN - rapidly progressive glo } \\
\text { merulonephritis; APACHEII - Acute Physiology and Chronic Health Evaluation; SAPS II - Simplifiec } \\
\text { Acute Physiology Score }\end{array}$} \\
\hline
\end{tabular}

encephalitis in 9.9\%; atypical hemolytic-uremic syndrome (HUS) in 7.9\%; Guillain-Barré syndrome in 4.9\%; acute polyradiculoneuropathy Guillain-Barré like in 4.9\%; chronic demyelinating polyradiculoneuropathy in 4.9\%; and thyrotoxic crisis in $4.0 \%$ procedures (Table I and Table II).

\section{TPE prescription and adjuvant therapies}

The mean number of TPE sessions per patient was $5.1 \pm 1.3$ (range: $3-9)$. Seven patients (35.0\%) required treatment every other day and the remaining underwent daily TPE. TPE dose was calculated according to the patient plasma volume and values ranged from $2500-3500 \mathrm{ml}$.

Human albumin ( $5 \%$ solution) was used as replacement fluid in most TPE procedures $(79.3 \%)$ and FFP in the rest. FFP was used in all patients with diagnosis of TTP and one patient with ANCA-associated vasculitis with RPGN needing dialysis (20.7\%).

The most frequently used vascular access was a hemodialysis catheter in the right jugular vein ( $51.5 \%$ of procedures). The other accesses 


\section{Table II}

Cohort description

\begin{tabular}{|c|c|c|c|c|c|c|c|c|}
\hline Patient & Age & Gender & Indication & $\begin{array}{c}\text { ASFA } \\
\text { Indication }\end{array}$ & $\begin{array}{c}\text { Treatments } \\
\text { number }\end{array}$ & APACHE II & Adjuvant immunosuppression & Evolution \\
\hline 1 & 26 & M & ANCA-associated RPGN with alveolar hemorrhage & 1 & 4 & 17 & Steroids & Favorable \\
\hline 2 & 83 & $\mathrm{~F}$ & Acute polyradiculoneuropathy & $I^{*}$ & 5 & 28 & Steroids & Unfavorable \\
\hline 3 & 57 & M & Atypical HUS & $\|$ & 4 & 17 & None & Favorable \\
\hline 4 & 41 & M & TTP & 1 & 6 & 7 & Steroids & Favorable \\
\hline 5 & 67 & $\mathrm{~F}$ & Guillain-Barré syndrome & 1 & 4 & 15 & Steroids + intravenous immunoglobulin & Unfavorable \\
\hline 6 & 40 & $\mathrm{~F}$ & Myasthenia gravis & 1 & 5 & 5 & Steroids & Unfavorable \\
\hline 7 & 21 & $\mathrm{~F}$ & Myasthenia gravis & 1 & 5 & 2 & Steroids & Favorable \\
\hline 8 & 31 & $\mathrm{~F}$ & Anti-NMDA receptor antibody encephalitis & 1 & 5 & 10 & Steroids + azathioprine & Favorable \\
\hline 9 & 41 & M & TTP & 1 & 6 & 8 & Steroids & Favorable \\
\hline 10 & 20 & $\mathrm{~F}$ & Immune complex RPGN & III & 4 & 21 & Mycophenolate mofetil & Favorable \\
\hline 11 & 57 & M & Atypical HUS & $\|$ & 6 & 20 & None & Unfavorable \\
\hline 12 & 34 & M & ANCA-associated RPGN with alveolar hemorrhage & 1 & 3 & 16 & Steroids & Unfavorable \\
\hline 13 & 40 & $\mathrm{~F}$ & Myasthenia gravis & 1 & 5 & 5 & Steroids & Favorable \\
\hline 14 & 20 & $\mathrm{~F}$ & Immune complex RPGN & III & 5 & 17 & Mycophenolate mofetil & Unfavorable \\
\hline 15 & 21 & $\mathrm{~F}$ & Myasthenia gravis & 1 & 5 & 2 & Steroids & Favorable \\
\hline 16 & 31 & $\mathrm{~F}$ & Anti-NMDA receptor antibody encephalitis & 1 & 6 & 14 & Steroids + azathioprine & Favorable \\
\hline 17 & 77 & M & Myasthenia gravis & 1 & 5 & 19 & Steroids + intravenous immunoglobulin & Favorable \\
\hline 18 & 64 & $\mathrm{~F}$ & Chronic demyelinating polyradiculoneuropathy & 1 & 5 & 6 & Steroids + intravenous immunoglobulin & Unfavorable \\
\hline 19 & 45 & $\mathrm{~F}$ & thyrotoxic crisis & III & 4 & 12 & None & Favorable \\
\hline 20 & 70 & M & ANCA-associated RPGN requiring RRT & 1 & 9 & 15 & Steroids + cyclophosphamide + rituximab & Unfavorable \\
\hline
\end{tabular}

* Assumed Guillain-Barré polyneuropathy like. M - male; F - female; ANCA - anti-neutrophil cytoplasmic antibody; APACHEll - Acute Physiology and Chronic Health Evaluation; HUS - hemolytic-uremic syndrome; TTP - thrombotic thrombocytopenic purpura; NMDA - anti-N-methyl D-aspartate; RPGN - rapidly progressive glomerulonephritis; RRT - Renal Replacement Therapy.

were hemodialysis catheters in the left femoral vein (21.8\%), right femoral vein (17.8\%) and in the left jugular vein (8.9\%). Anticoagulation with unfractionated heparin (UFH) was used in $63.4 \%$ of the treatments, and the remaining were conducted without anticoagulation.

Adjuvant immunosuppression was used in 17 (70.0\%) patients: $52.9 \%$ (9 patients) were treated only with steroid therapy and the remainder were treated with azathioprine ( 2 patients), intravenous immunoglobulin (3 patients) or cyclophosphamide plus rituximab (1 patient) in combination with steroids and TPE. Two patients were treated with mycophenolate mofetil in combination with TPE.

Twelve patients (60\%) required at least one organ support: $35.0 \%$ patients RRT; $50.0 \%$ invasive mechanical ventilation; and $15.0 \%$ vasopressor support. Half the patients needed antibiotic therapy during TPE technique.

\section{Complications and therapy outcomes}

A total of 46 (45.5\%) complications occurred in the 101 TPE sessions. On average, there were 0.46 complications per procedure and 2.1 per patient (range: $1-4$ ) during all TPE sessions.

The most frequent complications were transient hypotension $(6.9 \%$ of procedures) and hypocalcemia (4.9\%). Several infections were documented during the TPE period: urinary tract infections (3 patients), pneumonia ( 2 patients), and catheter-related infections (3 patients).
There were 4 (3.9\%) access-related complications: bleeding (1 patient) and infections (3 patients) (Table III).

Twenty-one (45.6\%) of the complications were classified as mild and $50.0 \%$ classified as moderate. The only severe complication was

\section{Table III}

Complications of therapeutic plasma exchange treatment

\begin{tabular}{l|c}
\multicolumn{1}{c|}{ Adverse event } & Procedures (\%) \\
\hline Hypotension & $7(6.9 \%)$ \\
Infection, non-catheter related & $5(4.9 \%)$ \\
Circuit clotting & $2(1.9 \%)$ \\
Symptoms & \\
\hline Muscle cramps & $3(2.9 \%)$ \\
\hline Nausea or vomiting & $2(1.9 \%)$ \\
\hline Paresthesia & $2(1.9 \%)$ \\
Access related complications & \\
\hline Infection, catheter & $3(2.9 \%)$ \\
\hline Bleeding, catheter & $1(0.9 \%)$ \\
\hline Abnormalities in laboratory test results & \\
\hline Anemia & $2(1.9 \%)$ \\
\hline Hyponatremia & $3(2.9 \%)$ \\
\hline Hypokalemia & $4(3.9 \%)$ \\
\hline Hypocalcemia & $5(4.9 \%)$ \\
\hline Hypomagnesemia & $1(1.9 \%)$ \\
\hline Hypofibrogenemia & $1(1.9 \%)$ \\
\hline
\end{tabular}


circuit clotting ( 2 procedures) which forced interruption of the treatment.

After plasmapheresis treatment, 12 patients $(60.0 \%)$ had a favorable outcome (Table I). Regarding the patients who had an unfavorable outcome (8 patients, 40.0\%): 4 remained dependent on hemodialysis after discharge from hospital (2 patients with ANCA-associated vasculitis, 1 patient with HUS, and 1 patient with immune complex RPGN); 1 patient with myasthenia gravis maintained the motor deficit observed on admission to the ICU; the patient with acute polyradiculoneuropathy maintained a motor deficit and need of tracheostomy after hospital discharge; the patient with Guillain-Barré syndrome and the patient with chronic demyelinating polyradiculoneuropathy died. There were no deaths related to the TPE procedure itself.

\section{DISCUSSION}

The introduction of TPE in clinical practice significantly reduced morbidity and mortality in patients with several pathologies, most importantly, thrombotic microangiopathies and myasthenia gravis ${ }^{12}$. Improvements in the technique over the last decade have helped minimize the complications of TPE treatments ${ }^{9,10}$.

This study aims to share an almost 20-year experience of TPE use in ICU patients. To our knowledge, this is the first study to analyze TPE in a Portuguese critically ill setting.

The most frequent indications for TPE in our study were myasthenia gravis, thrombotic microangiopathies and ANCA-associated vasculitis. Other critical care case series ${ }^{8,14-17}$ showed similar results, with thrombotic microangiopathies and nervous system disorders being the most frequent diagnosis of patients submitted to TPE. Although neurologic diseases are the most common reason for TPE in many ICU cases series, registries show a decrease in the number of TPE procedures over time, partially attributed to the decrease of TPE procedures performed in patients with Guillain-Barré syndrome ${ }^{10}$. Recent studies ${ }^{12,18,19}$ have shown similar outcomes between treatment with TPE or intravenous immunoglobulin in Guillain-Barré syndrome and many centers, like ours, started to use intravenous immunoglobulin as first-line therapy and reserve TPE for patients who do not respond to intravenous immunoglobulin ${ }^{10,14}$. In fact, only one patient with Guillain-Barré syndrome was treated with plasmapheresis in our study, in accordance with the most recent epidemiological data. The past years have seen a change in diseases in which we use TPE as therapy, with a stabilization in the number of neurological pathologies and a progressive increase in renal and hematological pathologies ${ }^{10,19}$. In addition, there has been a significant expansion in the number and diversity of ASFA indications for plasmapheresis ${ }^{6}$.

In our unit, TPE is performed by the filtration method, using the same machine and central venous catheters used for continuous RRT. Familiarization of health professionals with the equipment has rendered filtration TPE popular in the critical care setting ${ }^{1,2,20}$.

The selection of the replacement fluid varies according to the underlying disease ${ }^{2}$. Albumin solution was the most frequently replacement fluid used in our patients, as in other similar studies ${ }^{13,14,17,21}$. The main advantages of albumin are the low incidence of side effects, namely anaphylactic reactions, as opposed to FFP. Therefore, FFP is reserved for patients who need to replace plasmatic components during the technique $\mathrm{e}^{1,2}$.

TPE requires anticoagulation to prevent clots from forming in the extracorporeal circuit ${ }^{1,2,5}$. UFH was the most used anticoagulant used in our study. Higher doses are required compared to those used for continuous RRT, since heparin binds to plasma proteins and is lost with them during procedure 20,22 . We had an incidence of circuit clotting of $1.9 \%$, which occurred in patients who had contraindications for anticoagulation. The high number of patients without anticoagulation in our study can be explained by the fact that it is frequent for critical patients to have contra-indications, such as thrombocytopenia and high risk of bleeding, for heparin. Citrate was not available in our center.

The association of immunosuppressors in patients undergoing TPE is quite frequent, since most indications for the technique are immunological. Immunosuppression is needed to reduce the production of pathogenic antibodies and modulations of cell mediated immunity ${ }^{2}$. Almost all patients in our study were treated with immunosuppressors combined with plasmapheresis (70\%). Other ICU studies showed similar percentages of adjuvant immunosuppressive drugs use (76\%$-82 \%)^{8,11,13}$. The most used immunosuppressants were steroids, intravenous immunoglobulin, mycophenolate mofetil and azathioprine. Eculizumab has been associated with improvements in renal function and the interruption of plasma therapy and is currently recommended by several guidelines as the front-line therapy for HUS ${ }^{1,6}$. Eculizumab was not used in both cases of HUS in our population because it was not available at that time.

Our cohort was composed of critically ill patients with high illness severity scores and a high incidence of organ support. In $60 \%$ it was necessary to start at least one organ support (mechanical ventilation-50\%, RRT- $37.5 \%$ or vasopressor therapy-12.5\%) which is in accordance with a Spanish study of 24 ICU patients submitted to plasmapheresis where $62.5 \%$ needed organ support ${ }^{14}$. In contrast, Lemaire et al. ${ }^{8}$ and Benítez et al. ${ }^{14}$ showed a different incidence of organ support with $14-27.8 \%$ patients requiring vasopressor therapy, 22-31.6\% mechanical ventilation, and $24-26.3 \%$ patients requiring RRT. These differences are probably related to the underlying diseases, since our study included more patients with myasthenia gravis (5 vs. none ${ }^{8,13}$ ) which may explain the higher need of mechanical ventilation.

At present, TPE is considered a safe treatment ${ }^{1,11-14}$. In our study, adverse effects related to the technique were observed in $45.5 \%$ of the procedures but most (95.7\%) were mild or moderate. The incidence of complications reported in similar studies is quite variable. Bramlage et al. ${ }^{19}$ and Szczeklik et al. ${ }^{11}$ showed $11.1 \%$ of adverse events. Benitez et al. ${ }^{14}$ detected adverse effects during the technique in $65 \%$ of patients. The differences between the incidence of adverse events in these studies are probably related to the fact that intensive care patients have a variable complexity and severity. The definition of adverse effect is also an important factor when interpreting and comparing complication rates between studies. The most frequent adverse event observed in our study was hypotension (6.9\% of TPE procedures), 
which is comparable to other studies ${ }^{11,14,16}$. In our population most cases resolved spontaneously and there was no need to initiate vasopressors or suspend the technique. It is also important to note that only one hemorrhagic event $(0.9 \%$ of procedures) was observed despite the majority of procedures having been performed with UFH. A significant percentage of patients developed hypocalcemia $(4.9 \%$ of procedures) even without the use of anticoagulation with citrate, which is the most frequent cause of hypocalcemia ${ }^{1,2,9}$. This is in accordance with the literature, where hemorrhagic events are reported in $0.2-2.4 \%^{11,13,21}$ and hypocalcemia in $7.8-35.2 \%$ of the procedures ${ }^{11,14}$. We decided to register the infections developed during TPE therapy, but this study was not designed to allow us to establish a causal association between the incidence of infections and the use of TPE. There are many factors that may explain the occurrence of infections: (i) depletion of immunoglobulins; (ii) adjuvant immunosuppressive therapy; (iii) the complexity of ICU patients that are exposed to invasive procedures or devices. In our study infections were quite frequent (31.2\% of patients), similarly to a Chilean study of critically ill patients ${ }^{13}$ that showed infections in $25 \%$. TPE was discontinued two times $1.9 \%$ of procedures). Other studies showed a similar incidence of interruption of the technique due to complications $(0.12-1.35 \%)^{11,12,19}$.

Two patients of our study (10\%) died but deaths were attributed to the advanced stage of the underlying disease and not to the TPE technique itself. Other authors found similar or higher mortality rates, such as Szczeklik et al. ( $9 \%$ of mortality) $)^{11}$ or C. Benítez et al. (15\% of mortality $)^{13}$. TPE associated mortality is estimated at $0.1 \%{ }^{15}$.

In our population, similarly to the study by Szczeklik et al. ${ }^{11}$, most of the conditions that led to plasmapheresis were neurological. C. Benítez et al. reported some different results: $45 \%$ cases of TTP and $30 \%$ cases of vasculitis $^{13}$. In this study ${ }^{14}$, the number of patients requiring organ support and APACHEII on admission was similar to ours, which probably means that the underlying disease was an important factor contributing to the outcomes.

In our study, TPE proved to be an effective treatment, since most patients (82.5\%) had a favorable clinical evolution. Similarity, the 2005 International Apheresis Registry ${ }^{16}$ showed improvement of $74.8 \%$ of patients under TPE and in three critical care studies, a favorable clinical evolution was observed in $75 \% 14,15$ and $91 \%^{11}$, reinforcing the effectiveness of TPE as a therapeutic intervention in critically ill patients. It is difficult to interpret the role of TPE in patients whose clinical condition worsened under therapy, and it is possible that the advanced stage of the underlying disease influenced the unfavorable outcome.

The main limitations of this study are its retrospective nature and the small sample of patients with a high heterogeneity of pathologies. This last fact does not allow us to conduct a more extensive analysis among patients with the same pathology. Nevertheless, this is a handicap of most critical care TPE studies, due to the relatively low incidence of these procedures in the critical care setting. We provide the first comprehensive analysis of the TPE practice in a Portuguese ICU.

In our critical care population, TPE was an effective and safe therapy, as observed in similar studies. The progressive improvement of monitoring and implementation of prevention measures by experienced teams is essential to avoid complications. Careful patient selection for TPE is also important to optimize technique effectiveness and safety.

Our study, in characterizing a Portuguese critical care unit experience with TPE, contributes to the accumulated knowledge and consequently helps to improve best practices worldwide.

Disclosure of potential conflicts of interest: none declared

\section{References}

1. Daga Ruiz D, Fonseca San Miguel F, González de Molina FJ, Úbeda-Iglesias A, Navas Pérez A, Jannone Forés $R$. Plasmapheresis and other extracorporeal filtration techniques in critical patients. Med Intensiva. 2017;41(3):174-187.

2. Daugirdas J, Blake P, Ing T. Handbook of Dialysis. 2014.

3. Nakanishi T, Suzuki N, Kuragano T, Nagasawa Y, Hasuike Y. Current topics in therapeutic plasmapheresis. Clin Exp Nephrol. 2014;18(1):41-49.

4. Williams ME, Balogun RA. Principles of separation: Indications and therapeutic targets for plasma exchange. Clin J Am Soc Nephrol. 2014;9(1):181-190.

5. Madore F. Plasmapheresis technical aspects and indications. Crit Care Clin. 2002;18(2):375-392.

6. Padmanabhan A, Connelly-Smith L, Aqui N, Balogun RA, Klingel R, Meyer E, et al. Guidelines on the Use of Therapeutic Apheresis in Clinical Practice - Evidence-Based Approach from the Writing Committee of the American Society for Apheresis: The Eighth Special Issue. Journal of clinical apheresis. 2019;34(3):171-354.

7. Nguyen TC, Kiss JE, Goldman JR, Carcillo JA. The Role of plasmapheresis in critical illness. Crit Care Clin 2012;28(3):453-468.

8. Lemaire A, Parquet N, Galicier L, Boutboul D, Bertinchamp R, Malphettes M, et al. Plasma exchange in the intensive care unit: technical aspects and complications. J Clin Apher. 2017;32(6):405-412.

9. Mokrzycki MH, Balogun RA. Therapeutic apheresis: a review of complications and recommendations for prevention and management. J Clin Apher. 2011;26:243-248.

10. Norda R, Stegmayr BG, Berlin G, Kurkus J, Jonsson S, Söderström T, et al. Therapeutic apheresis in Sweden: update of epidemiology and adverse events. Transfus Apher Sci. 2003;29(2):159-166.

11. Szczeklik W, Wawrzycka K, Włudarczyk A, Sega A, Nowak I, Seczyńska B, et al. Complications in patients treated with plasmapheresis in the intensive care unit. Anaesthesiol Intensive Ther. 2013;45(1):7-13.

12. Schwartz J, Padmanabhan A, Aqui N, Balogun RA, Connelly-Smith L, Delaney M, et al. Guidelines on the Use of Therapeutic Apheresis in Clinical Practice-Evidence-Based Approach from the Writing Committee of the American Society for Apheresis: The Seventh Special Issue. J Clin Apher. 2016;31:149-162.

13. Basic-Jukic N, Kes P, Glavas-Boras S, Brunetta B, Bubic-Filipi L, Puretic Z. Complications of therapeutic plasma exchange: experience with 4857 treatments. Ther Apher Dial. 2005;9(5):391-395.

14. Benitez C, Andresen M, Farias G, Castillo C, Henriquez M, Pereira J. Indications, adverse effects and results of plasmapheresis in critical care patients. Rev Med Chil. 2005;133:1441-1448.

15. Salazar Ramirez C, Daga Ruiz D, Cota Delgado F, Fernández Aguirre C, Fernández Añon JM, Garcia Fernández JM. Utilidad de la plasmaferesis en cuidados intensivos. Med Intensiva. 2010;34(1):7478.

16. Sengul Samanci N, Ayer M, Gursu M, Ar MC, Yel K, Ergen A, et al. Patients treated with therapeutic plasma exchange: a single center experience. Transfus Apher Sci. 2014;51(3):83-89.

17. Malchesky PS, Koo AP, Skibinski Cl, Hadsell AT, Rybicki LA. Apheresis technologies and clinical applications: the 2007 international apheresis registry. Ther Apher Dial. 2010;14(1):52-73.

18. Hughes RAC, Wijdicks EFM, Barohn R, Benson E, Cornblath DR, Hahn AF. Practice parameter: Immunotherapy for Guillain - Barré syndrome Report of the Quality Standards Subcommittee of the American Academy of Neurology. Neurology. 2003;61:736-740.

19. Brunetta Gavranić B, Bašić-Jukić N, Kes P. Changes in indications for therapeutic plasma exchange over the last 27 years in Croatia. Ther Apher Dial. 2011;15(6):587-592.

20. Gashti CN, Andreoli DC, Patel D. Membrane-based therapeutic plasma exchange (mTPE): technical and clinical experience. J Clin Apher. 2018;33:38-45.

21. Bramlage CP, Schroder K, Bramlage P, Ahrens K, Zapf A, Muller GA, et al. Predictors of complications in therapeutic plasma exchange. J Clin Apher. 2009;24:225-231.

22. Lee $G$, Arepally GM. Anticoagulation techniques in apheresis: from heparin to citrate and beyond. J Clin Apher. 2012;27:117-125.

\section{Correspondence to:}

Rita Calça, MD

Nephrology Department, Hospital de Santa Cruz

Centro Hospitalar Lisboa Ocidental

Av. Prof. Dr. Reinaldo dos Santos 27-29, 2790-134, Carnaxide, Portugal.

E-mail: arrcalca@gmail.com 